\title{
Quantum Valley Hall Effect, Orbital Magnetism, and Anomalous Hall Effect in Twisted Multilayer Graphene Systems
}

\author{
Jianpeng Liu, ${ }^{1}$ Zhen $\mathrm{Ma},{ }^{2}$ Jinhua Gao, ${ }^{2}$ and Xi Dai ${ }^{1}$ \\ ${ }^{1}$ Department of Physics, Hong Kong University of Science and Technology, Kowloon, Hong Kong \\ ${ }^{2}$ School of Physics, Huazhong University of Science and Technology, Wuhan 430074, China
}

(Received 7 April 2019; revised manuscript received 10 May 2019; published 8 August 2019)

\begin{abstract}
We study the electronic structures and topological properties of $(M+N)$-layer twisted graphene systems. We consider the generic situation that $N$-layer graphene is placed on top of the other $M$-layer graphene and is twisted with respect to each other by an angle $\theta$. In such twisted multilayer graphene systems, we find that there exist two low-energy flat bands for each valley emerging from the interface between the $M$ layers and the $N$ layers. These two low-energy bands in the twisted multilayer graphene system possess valley Chern numbers that are dependent on both the number of layers and the stacking chiralities. In particular, when the stacking chiralities of the $M$ layers and $N$ layers are opposite, the total Chern number of the two low-energy bands for each valley equals $\pm(M+N-2)$ (per spin). If the stacking chiralities of the $M$ layers and the $N$ layers are the same, then the total Chern number of the two low-energy bands for each valley is $\pm(M-N)$ (per spin). The valley Chern numbers of the low-energy bands are associated with large, valley-contrasting orbital magnetizations, suggesting the possible existence of orbital ferromagnetism and anomalous Hall effect once the valley degeneracy is lifted either externally by a weak magnetic field or internally by Coulomb interaction through spontaneous symmetry breaking. Such an orbital ferromagnetic state is characterized by chiral current loops circulating around the $A A$ region of the moiré pattern, which can be experimentally detected.
\end{abstract}

\section{INTRODUCTION}

Twisted bilayer graphene (TBG) has drawn significant attention recently due to the observations of the correlated insulating phases [1-5] and unconventional superconductivity [5-7]. At small twist angles, the low-energy states of TBG are characterized by four low-energy bands contributed by the two nearly decoupled monolayer valleys $[8,9]$. Around the "magic angles," the bandwidths of the four low-energy bands become vanishingly small, and these nearly flat bands are believed to be responsible for most of those exotic properties observed in TBG. Numerous theories have been proposed to understand the electronic structures [10-20], the correlated insulating phase $[10,21-31]$, and the mechanism of superconductivity $[10,22,24,25,27,32-38]$.

On the other hand, interesting topological features have already emerged in the electronic structure of TBG. It has been shown that the four low-energy bands are topologically nontrivial in the sense that they are characterized by

Published by the American Physical Society under the terms of the Creative Commons Attribution 4.0 International license. Further distribution of this work must maintain attribution to the author(s) and the published article's title, journal citation, and DOI. odd windings of Wilson loops [14,18,39], which is an example of the fragile topology [15]. The four flat bands have been further proposed to be equivalent to the zeroth pseudo-Landau levels (LLs) with opposite Chern numbers and sublattice polarizations [18], which is the origin of the nontrivial band topology in the TBG system.

Recently, unconventional ferromagnetic superconductivity and the correlated insulating phase have been observed in twisted double bilayer graphene [40-42] and trilayer graphene moiré systems [43-45]. It implies that the lowenergy flat bands, which are believed to be responsible for the correlated physics in TBG, may also exist in the twisted double bilayer graphene system. A recent theoretical study indeed revealed the presence of flat bands in twisted double bilayer graphene [46]. Motivated by these works, in this paper we study the electronic structures and topological properties of twisted multilayer graphene (TMG). In particular, we consider the most generic situation, that the $N$-layer chirally stacked graphene is placed on top of the other $M$-layer chirally stacked graphene and they are twisted with respect to each other by a nonvanishing angle $\theta$, as schematically shown in Fig. 1(a) (for the case of $M=2, N=2)$. In such an $(M+N)$-layer TMG system, we propose that there always exist two low-energy bands (for each valley), and that the bandwidths of the two lowenergy bands become vanishingly small at the magic angles 
(a)

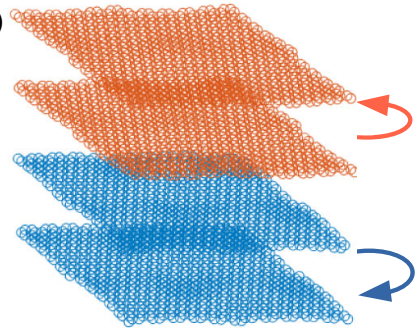

(b)

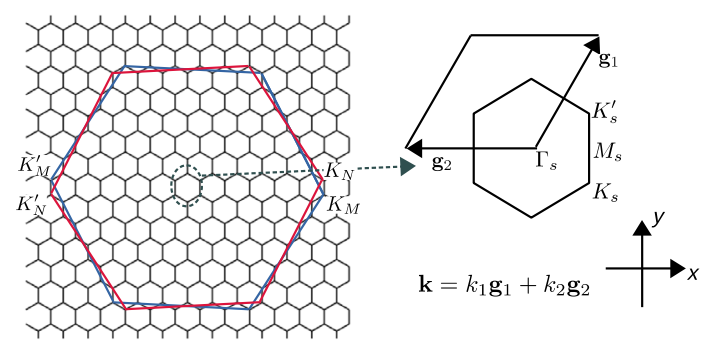

FIG. 1. (a) Left: Structure of the twisted multilayer graphene with $M=2$ and $N=2$ (see text). Right: Moiré pattern of the twisted multilayer graphene, seen from the top. $\mathbf{t}_{1}$ and $\mathbf{t}_{2}$ denote the lattice vectors of the moiré supercell. (b) The Brillouin zones of the top $N$ multiplayers, bottom $M$ multilayers, and the moiré supercell are plotted in red, blue, and black lines, respectively.

of twisted bilayer graphene for arbitrary numbers of layers $M$ and $N$. The flat bands in the TMG system can be interpreted from the pseudo-LL representation of TBG [18], and are protected by an approximate chiral symmetry in chiral graphene multilayers.

Moreover, we also find that there is a Chern-number hierarchy in the $(M+N)$-layer TMG system. In particular, when the stacking chiralities of the $M$ layers and $N$ layers are the same, the total Chern number of the two low-energy flat bands for each monolayer valley equals $\pm(M-N)$ for each spin species [47]. On the other hand, if the stacking chiralities of the $M$ layers and the $N$ layers are opposite, then the total Chern number of the two low-energy bands for each valley is $\pm(M+N-2)$. Taking the twisted double bilayer graphene $(M=2, N=2)$ as an example, when the bottom bilayer and the top bilayer are both $A B$ or $B A$ stacked (same stacking chirality), the total Chern number of the two low-energy flat bands would be zero; on the other hand, if the bottom bilayer is $A B(B A)$ stacked and the top bilayer is $B A(A B)$ stacked (opposite stacking chiralities), then the total Chern number of the two low-energy flat bands for each valley equals \pm 2 . The valley Chern numbers can be further tuned by an external electric field, leading to the gate-tunable quantum valley Hall effect.

The nonzero valley Chern numbers of the low-energy flat bands are characterized by large and valley-contrasting orbital magnetizations. With the presence of an external magnetic field or the spontaneous symmetry breaking induced by the Coulomb interactions, the valley degeneracy is expected to be broken, and a valley-polarized (quantum) anomalous Hall state may be realized. The valley polarized state is associated with chiral current loops, which generate local magnetic fields peaked at the $A A$ region. The local magnetic fields generated by the chiral current loops may be a robust experimental signature for the nonzero valley Chern number and the valley-polarized state in the TMG system. The flat bands at the universal magic angles, together with the Chern-number hierarchy and orbital magnetism, make the TMG systems a unique platform to study strongly correlated physics with nontrivial band topology, and may have significant implications on the observed ferromagnetic superconductivity and correlated insulating phase in twisted double bilayer graphene [41,42].

\section{ELECTRONIC STRUCTURES OF THE TWISTED MULTILAYER GRAPHENE SYSTEMS}

\section{A. Lattice structures}

We consider the most generic case of chirally stacked twisted multilayer graphene; i.e., we place $N$ chiral graphene multilayers on top of $M$ chiral graphene multilayers and twist them with respect to each other by an angle $\theta$. This is schematically shown in Fig. 1(a) for the case of $M=2, N=2$. Similar to the case of TBG, commensurate moiré supercells are formed when the twist angle $\theta(m)$ obeys the condition $\cos \theta(m)=\left(3 m^{2}+3 m+1 / 2\right) /\left(3 m^{2}+\right.$ $3 m+1)$ [48], where $m$ is a positive integer. The lattice vectors of the moiré superlattice are expressed as $\mathbf{t}_{1}=$ $\left(-\sqrt{3} L_{s} / 2, L_{s} / 2\right)$ and $\mathbf{t}_{2}=\left(0, L_{s}\right)$, where $L_{s}=\left|\mathbf{t}_{1}\right|=$ $a /[2 \sin (\theta / 2)]$ is the size of the moire supercell and $a=2.46 \AA$ is the lattice constant of graphene. In TBG it is well known that there are atomic corrugations, i.e., the variation of interlayer distances on the moire length scale. In particular, in the $A B(B A)$ region of TBG, the interlayer distance $d_{A B} \approx 3.35 \AA$, while in the $A A$-stacked region the interlayer distance $d_{A A} \approx 3.6 \AA$ [49]. Such atomic corrugations may be modeled as [12]

$$
d_{z}(\mathbf{r})=d_{0}+2 d_{1} \sum_{j=1}^{3} \cos \left(\mathbf{g}_{j} \cdot \mathbf{r}\right),
$$

where $\mathbf{g}_{1}, \mathbf{g}_{2}$, and $\mathbf{g}_{3}=\mathbf{g}_{1}+\mathbf{g}_{2}$ are the three reciprocal lattice vectors of the moirè supercell. We take $d_{0}=3.433 \AA$ and $d_{1}=0.0278 \AA$ in order to reproduce the interlayer distances in $A A$ - and $A B$-stacked bilayer graphene. In this paper, the atomic corrugations of the two twisted layers at the interface (between the $M$ layers and the $N$ layers) is also modeled by Eq. (1). On the other hand, the interlayer distances within the untwisted $M$ layers and the untwisted $N$ layers are set to the interlayer distance of Bernal bilayer graphene $d_{A B}=3.35 \AA$. At a small twist angle $\theta$, the Brillouin zone (BZ) of the moire supercell has been significantly reduced compared with those of the untwisted multilayers, as shown in Fig. 1(b). 


\section{B. Effective Hamiltonian}

The low-energy effective Hamiltonian of the twisted $(M+N)$-layer TMG of the $K$ valley is expressed as

$$
H_{\alpha, \alpha^{\prime}}^{K}(M+N)=\left(\begin{array}{cc}
H_{\alpha}^{K}(M) & \mathbb{U} \\
\mathbb{U}^{\dagger} & H_{\alpha^{\prime}}^{K}(N)
\end{array}\right),
$$

where $H_{\alpha}^{K}(M)$ and $H_{\alpha^{\prime}}^{+}(N)$ are the effective Hamiltonians for the $M$-layer and $N$-layer graphene with stacking chiralities $\alpha, \alpha^{\prime}=+/-$. In particular,

$$
H_{\alpha}^{K}(M)=\left(\begin{array}{ccccc}
h_{0}(\mathbf{k}) & h_{\alpha} & 0 & 0 & \cdots \\
h_{\alpha}^{\dagger} & h_{0}(\mathbf{k}) & h_{\alpha} & 0 & \cdots \\
0 & h_{\alpha}^{\dagger} & h_{0}(\mathbf{k}) & h_{\alpha} & \cdots \\
& & & \cdots &
\end{array}\right) \text {, }
$$

where $h_{0}(\mathbf{k})=-\hbar v_{F}\left(\mathbf{k}-\mathbf{K}_{M}\right) \cdot \boldsymbol{\sigma}$ stands for the lowenergy effective Hamiltonian for monolayer graphene near the Dirac point $\mathbf{K}_{M}$, and $h_{\alpha}$ is the interlayer hopping, with

$$
h_{+}=\left(\begin{array}{cc}
0 & 0 \\
t_{\perp} & 0
\end{array}\right),
$$

and $h_{-}=h_{+}^{\dagger}$.

The off-diagonal term $\mathbb{U}$ represents the coupling between the twisted $M$ layers and $N$ layers. Here we assume that there is only the nearest-neighbor interlayer coupling; i.e., the topmost layer of the $M$-layer graphene is only coupled with the bottom-most layer of the $N$-layer graphene. Thus,

$$
\mathbb{U}=\left(\begin{array}{ccc}
0 & \cdots & 0 \\
\vdots & \cdots & 0 \\
U(\mathbf{r}) e^{-i \Delta \mathbf{K} \cdot \mathbf{r}} & \cdots & 0
\end{array}\right),
$$

where the $2 \times 2$ matrix $U$ describes the tunneling between the Dirac states of the twisted bilayers $[9,12]$,

$$
U(\mathbf{r})=\left(\begin{array}{cc}
u_{0} g(\mathbf{r}) & u_{0}^{\prime} g\left(\mathbf{r}-\mathbf{r}_{A B}\right) \\
u_{0}^{\prime} g\left(\mathbf{r}+\mathbf{r}_{A B}\right) & u_{0} g(\mathbf{r})
\end{array}\right),
$$

where $\mathbf{r}_{A B}=\left(\sqrt{3} L_{s} / 3,0\right), u_{0}^{\prime}$ and $u_{0}$ denote the intersublattice and intrasublattice interlayer tunneling amplitudes, with $u_{0}^{\prime} \approx 0.098 \mathrm{eV}$, and $u_{0} \approx 0.078 \mathrm{eV}$ [12]. $u_{0}$ is smaller than $u_{0}^{\prime}$ due to the effects of atomic corrugations $[12,18]$. $\Delta \mathbf{K}=\mathbf{K}_{N}-\mathbf{K}_{M}=\left(0,4 \pi / 3 L_{s}\right)$ is the shift between the Dirac points of the $N$ layers and the $M$ layers. The phase factor $g(\mathbf{r})$ is defined as $g(\mathbf{r})=\sum_{j=1}^{3} e^{i \mathbf{q}_{j} \cdot \mathbf{r}}$, with $\mathbf{q}_{1}=\left(0,4 \pi / 3 L_{s}\right), \mathbf{q}_{2}=\left(-2 \pi / \sqrt{3} L_{s},-2 \pi / 3 L_{s}\right)$, and $\mathbf{q}_{3}=$ $\left(2 \pi / \sqrt{3} L_{s},-2 \pi / 3 L_{s}\right)$. It is worth noting that Eq. (2) is the effective Hamiltonian for the $K$ valley. The Hamiltonian for the $K^{\prime}$ valley is readily obtained by applying a timereversal operation to $H_{\alpha, \alpha^{\prime}}^{K}(M+N)$.

\section{Emergence of two flat bands and the universal magic angles}

We continue to study the electronic structures of the $(M+N)$-layer TMG systems using the effective Hamiltonian given by Eq. (2). The band structures for $(M=2, N=2),(M=2, N=4),(M=3, N=3)$, and $(M=3, N=4)$ at the first magic angle of TBG $\theta=1.05^{\circ}$ with the same stacking chiralities $\left(\alpha=\alpha^{\prime}=+\right)$ are shown in Figs. 2(a)-2(d), respectively [50]. Clearly there are two low-energy flat bands marked by the red lines that are separated from the other bands. The two low-energy bands are almost exactly flat at $\theta=1.05^{\circ}$ for all these TMG systems with different layers, indicating that the magic angle of TBG is universal for the TMG systems regardless of the number of layers. It turns out that the two flat bands in TMG originate from the twisted bilayer at the interface, and they remain flat even after being coupled with the other graphene layers due to an (approximate) chiral symmetry of Eq. (2). More details about the origin of the flat bands in the TMG systems can be found in Appendix A.

In realistic situations there are also further neighbor interlayer hoppings in graphene multilayers, which would break the chiral symmetry of the effective Hamiltonian in Eq. (2), and the flat bands shown in Fig. 2 would become more dispersive. In order to test the robustness of the flat bands, we have included all the second-neighbor and third-neighbor interlayer hoppings with intersite distances equal to $\sqrt{a^{2} / 3+d_{A B}^{2}}$ and $\sqrt{a^{2}+d_{A B}^{2}}$, respectively ( $d_{A B} \approx 3.35 \AA$ is the interlayer distance), and their amplitudes are denoted by $t_{2}$ and $t_{3}$. After including these
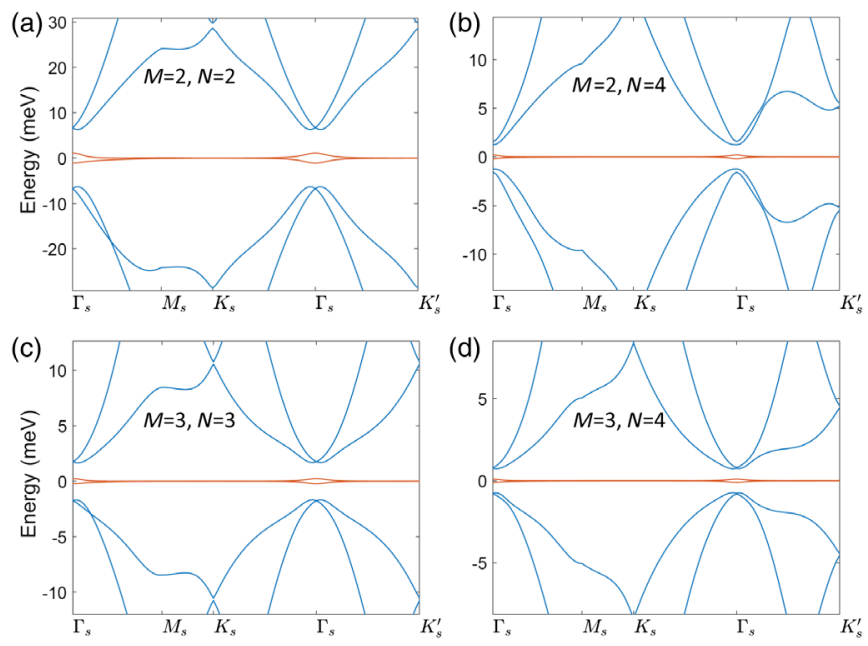

FIG. 2. The band structures of $(M+N)$-layer twisted multilayer graphene at the first magic angle $\theta=1.05^{\circ}$. (a) $M=2$, $N=2$, (b) $M=2, N=4$, (c) $M=3, N=3$, and (d) $M=3$, $N=4$. 

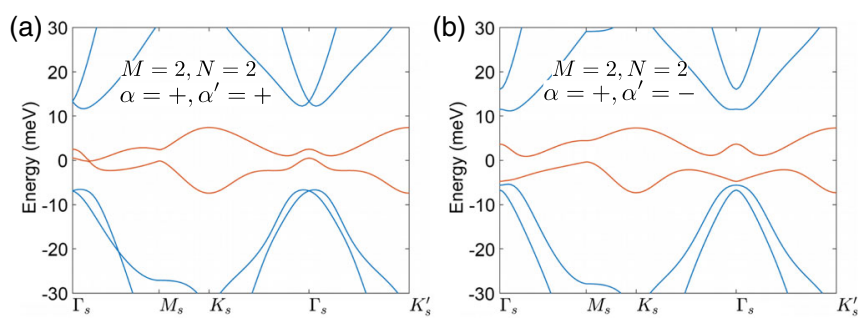

FIG. 3. The band structures of $(2+2)$-layer twisted multilayer graphene at the first magic angle $\theta=1.05^{\circ}$ with the more realistic interlayer hopping Eq. (7). (a) The two bilayers have the same stacking chirality, and (b) the two bilayers have opposite stacking chiralities.

terms, the interlayer hopping term with + stacking chirality becomes

$$
h_{+}=\left(\begin{array}{cc}
t_{2} f(\mathbf{k}) & t_{2} f^{*}(\mathbf{k}) \\
t_{\perp}-3 t_{3} & t_{2} f(\mathbf{k})
\end{array}\right)
$$

where in $t_{2}=0.21 \mathrm{eV}, t_{3} \approx 0.05 \mathrm{eV}$ are extracted from the Slater-Koster hopping parameters [see Eq. (9)]. In order to be consistent with the choice of $t_{2}$ and $t_{3}$, we set $t_{\perp}=0.48 \mathrm{eV}$, which is also from the Slater-Koster formula [Eq. (9)]. The phase factor $f(\mathbf{k})=\left(e^{-i \sqrt{3} a k_{y} / 3}+\right.$ $\left.\left.e^{i\left(k_{x} a / 2+\sqrt{3} a k_{y} / 6\right.}\right)+e^{i\left(-k_{x} a / 2+\sqrt{3} a k_{y} / 6\right.}\right)$. The interlayer hopping with - stacking chirality $h_{-}=h_{+}^{\dagger}$. The band structures of $(2+2)$-layer TMG at $\theta=1.05^{\circ}$ with the new interlayer hopping term Eq. (7) are shown in Fig. 3, where panels (a) and (b) denote the cases with the same and opposite stacking chiralities, respectively. Clearly, the two low-energy bands marked by the red lines become more dispersive due to the presence of the further-neighbor interlayer hoppings, but the bandwidths are still small $\sim 10-15 \mathrm{meV}$, and the two low-energy bands are still separated from the high-energy bands.

\section{CHERN-NUMBER HIERARCHY AND QUANTUM VALLEY HALL EFFECT}

\section{A. Chern-number hierarchy}

The flat bands at the universal magic angle make the TMG systems a perfect platform to study the strongly correlated physics. In addition to the flat bands and the universal magic angles, the low-energy bands in the TMG systems also exhibit unusual topological properties with nonvanishing valley Chern numbers. To be specific, when the stacking chiralities of the $M$ layers and the $N$ layers are the same, the total Chern number of the two low-energy bands for each monolayer valley equals $\pm(M-N)$. On the other hand, if the stacking chiralities of the $M$ layers and the $N$ layers are opposite, then the total Chern number of the two flat bands for each valley equals $\pm(M+N-2)$. Such a Chern-number hierarchy is more concisely summarized in the following equation:

$$
\begin{aligned}
& C_{\alpha, \alpha^{\prime}}^{K}=+\left[\alpha(M-1)-\alpha^{\prime}(N-1)\right], \\
& C_{\alpha, \alpha^{\prime}}^{K^{\prime}}=-\left[\alpha(M-1)-\alpha^{\prime}(N-1)\right],
\end{aligned}
$$

where $C_{\alpha, \alpha^{\prime}}^{K}\left(C_{\alpha, \alpha^{\prime}}^{K^{\prime}}\right)$ denotes the total Chern number of the two low-energy flat bands for the $K\left(K^{\prime}\right)$ valley, and the subscripts $\alpha, \alpha^{\prime}= \pm$ represent the stacking chiralities of the $M$ layers and $N$ layers. We would like to emphasize that the total Chern number of the two flat bands (per valley per spin) is a more robust quantity than the Chern number of each individual flat band. This is because the former is protected by the energy gaps between the two flat bands and the other high-energy bands, while the latter is crucially dependent on how the gap between the two flat bands is opened up.

In order to understand the Chern-number hierarchy of Eq. (8), we first divide the $(M+N)$-layer TMG system into three mutually decoupled subsystems: the TBG at the interface, the $(M-1)$ graphene monolayers below the interface TBG, and the $(N-1)$ graphene monolayers above the interface TBG, which are schematically shown in Fig. 5(a). We introduce a scaling parameter $0 \leq \lambda \leq 1$, and let the coupling strength between the three subsystems $t_{\perp} \rightarrow \lambda t_{\perp}$. We adiabatically turn on the coupling between the three subsystems by increasing $\lambda$ from 0 to 1 , then inspect the evolution of the band structures of the TMG system.

In Fig. 5(b), we show the band structure of $(3+2)$-layer TMG (of the $K$ valley) at $\theta=1.05^{\circ}$ with the scaling parameter $\lambda=0$. When $\lambda=0$, the magic-angle TBG at the interface would give rise to two flat bands with total Chern number 0, as marked by the red lines in Fig. 5(a). The $(M-1)$ graphene monolayers below the TBG interface would contribute two low-energy bands with dispersions $\sim \pm|\mathbf{k}|^{M-1}$ around $K_{s}$ [51]. Similarly, the $(N-1)$ layers above the TBG interface would contribute two lowenergy bands with dispersions $\sim \pm|\mathbf{k}|^{N-1}$ around $K_{s}^{\prime}$. Since we have considered the case $M=3$ and $N=2$, there are quadratic band touching at $K_{s}$ and linear band touching at $K_{s}^{\prime}$ in Fig. 5(b). More importantly, the bands with quadratic and linear dispersions are degenerate with the flat bands at $K_{s}$ and $K_{s}^{\prime}$ points for $\lambda=0$, which are classified as "singular flat bands" according to Ref. [52]. Such singular flat bands may acquire nonvanishing Chern numbers once the degeneracy at the band-crossing point is lifted.

If $\lambda$ becomes nonzero, gaps would be opened up at $K_{s}$ and $K_{s}^{\prime}$ for the bands with $\pm|\mathbf{k}|^{M-1}$ and $\pm|\mathbf{k}|^{N-1}$ dispersions, as shown in Fig. 5(c) for $\lambda=0.05$. As a result, the loop integral for the Berry connection of the conduction and valance bands around a loop enclosing the $K_{s}$ point would acquire the same Berry phase of $-\alpha(M-1) \pi[\alpha$ is the stacking chirality of the $(M-1)$ layers] and contribute $-\alpha(M-1) / 2$ to the total Chern number, respectively, which adds up to $-\alpha(M-1)$ (see Appendix B). On the 
other hand, the conduction and valence bands around the $K_{s}^{\prime}$ point contributed by the $(N-1)$ layers above the interface would acquire the same Berry phase of $\alpha^{\prime}(N-1) \pi\left[\alpha^{\prime}\right.$ is the stacking chirality of the $(N-1)$ layers], with the total Chern number of $\alpha^{\prime}(N-1)$ (see Appendix B). It is well known that the total Chern number of the bands from the $(M-1)$ and $(N-1)$ layers must cancel that of the two flat bands from the interface TBG; it follows that the total Chern number of the two flat bands for the $K$ valley equals $\alpha(M-1)-\alpha^{\prime}(N-1)$. As $\lambda$ is further increased, the conduction and valence bands from the $(M-1)$ and $(N-1)$ layers are further pushed to high energies, as shown in Fig. 5(d), for $\lambda=0.5$, and the Chern number of the two flat bands would remain unchanged. Thus, Eq. (8) has been proved. See Appendix B for more details.

Equation (8) has been numerically verified using the effective Hamiltonian of TMG shown in Eq. (2). In particular, in Fig. 4(a) we plot the Wilson-loop eigenvalues [denoted as $w(k)$ ] of the $(2+2)$ TMG $(M=2, N=2)$ at the first magic angle with the same stacking chirality $\left(\alpha=\alpha^{\prime}=+\right)$. The red diamonds and blue circles represent the Wilson loops of the $K$ and $K^{\prime}$ valleys, respectively. As clearly shown in the figure, for each valley the total Chern number of the two flat bands vanishes. In Fig. 4(b) we plot the Wilson loops of the $(2+2)$ TMG at the first magic angle, but with opposite stacking chiralities $(\alpha=+$, $\alpha^{\prime}=-$ ). It is clearly seen that for the $K$ valley (blue circles) the two Wilson loops carry the same Chern number +1 , giving rise to a total Chern number of +2 for the $K$ valley ( -2 for the $K^{\prime}$ valley), which is consistent with Eq. (8). In Figs. 4(c) and 4(d) we plot the Wilson-loop eigenvalues for
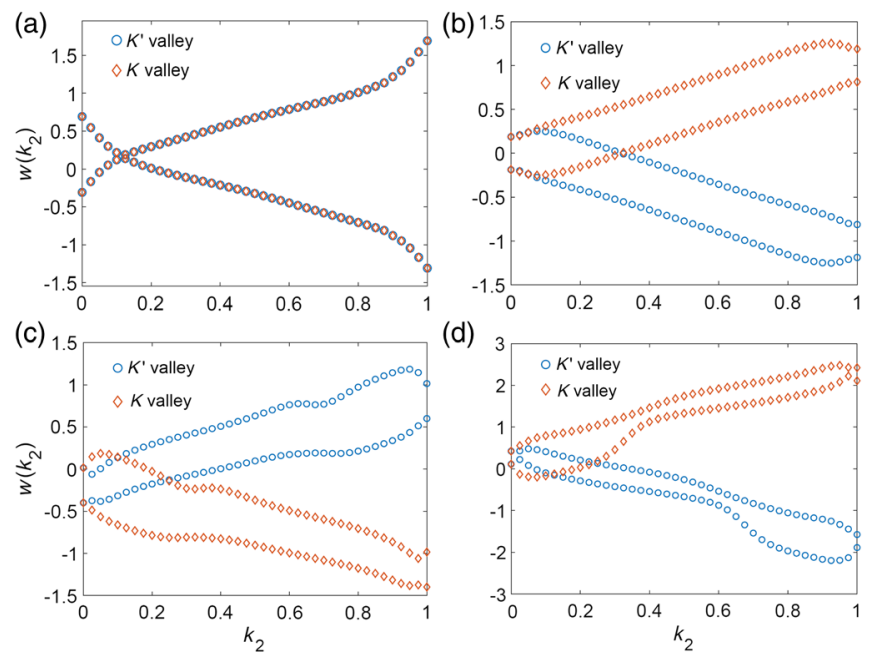

(d)

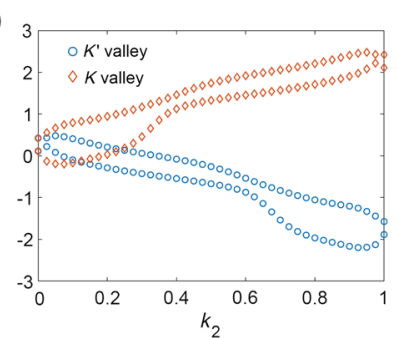

FIG. 4. The Wilson loops of $(M+N)$-layer twisted multilayer graphene at the first magic angle $\theta=1.05^{\circ}$. (a) $M=2, N=2$ with the same stacking chiralities, (b) $M=2, N=2$, with opposite stacking chiralities, (c) $M=2, N=4$, with the same stacking chiralities, and (d) $M=2, N=4$, with opposite stacking chiralities. the $(2+4)$ TMG $(M=2, N=4)$ at the first magic angle. When the stacking chiralities are the same, the total Chern number of the two flat bands for the $K\left(K^{\prime}\right)$ valley equals $-2(+2)$; while if the stacking chiralities are opposite, then the total Chern number of the two flat bands equals $+4(-4)$ for the $K\left(K^{\prime}\right)$ valley. Again, this is in perfect agreement with Eq. (8). We have also numerically tested the other $(M+N)$-layer TMG with $M, N$ extending from 1 to 5, and they are all consistent with Eq. (8).

We have also considered the more realistic situation in which the interlayer hopping is given by Eq. (7) instead of Eq. (4). Since the chiral symmetry is broken in Eq. (7), the Chern-number hierachy given by Eq. (8) is no longer exact. However, since the Chern number of concern is the total Chern number of the two low-energy flat bands, it should remain unchanged as long as the two low-energy flat bands remain separated from the other high-energy bands. We have numerically calculated the total valley Chern numbers of the two low-energy bands in $(M+N)$-layer TMG systems $(M, N$ varies from 1 to 5$)$ at $\theta=1.05^{\circ}$ using the more realistic interlayer hopping term Eq. (7), and find that the Chern-number hierarchy of Eq. (8) remains correct for the cases of $(M=1, N=2),(M=1, N=3)$, $(M=1, N=4), \quad(M=1, N=5), \quad(M=2, N=2)$, $(M=2, N=3)$, and is partially correct (for one stacking configuration) for $(M=3, N=3),(M=2, N=4)$, and $(M=3, N=5)$.

\section{B. Gate-tunable quantum valley Hall effect}

The valley Chern numbers given by Eq. (8) can be further tuned by applying a vertical electric potential $V_{\perp}$. Taking the case of $(2+2)$-layer TMG and $(2+1)$-layer TMG as an example, we study the dependence of the valley Chern numbers of each of the two low-energy bands on the vertical electric potential $V_{\perp}$ at $\theta=1.05^{\circ}$. The valley Chern number of each band is denoted by $C_{n \alpha \alpha^{\prime}}^{K}$, with the band index $n=1,2$, the stacking chirality $\alpha, \alpha^{\prime}= \pm$, and the valley index $K$.

In Table I we show the dependence of $C_{1 \alpha \alpha^{\prime}}^{K}, C_{2 \alpha \alpha^{\prime}}^{K}$ on the vertical electric potential $V_{\perp}$ (in units of $\mathrm{meV}$ ) for $(2+2)$ layer TMG. The valley Chern numbers are calculated using the effective Hamiltonian Eq. (2) with the more realistic interlayer hopping Eq. (7). When the stacking chiralities are the same (both + ), the Chern number of each of the two low-energy bands becomes \pm 3 once a small $V_{\perp} \sim 12 \mathrm{meV}$ is applied, whereas the total Chern number of the two bands still sums to zero. As $\left|V_{\perp}\right|$ increases, the valley Chern numbers of the two flat bands are changed to \pm 2 at $\left|V_{\perp}\right|=24 \mathrm{meV}$, then become 0 and \pm 1 , respectively, at $\left|V_{\perp}\right|=36 \mathrm{meV}$, and both become \pm 1 when $\left|V_{\perp}\right| \gtrsim 48 \mathrm{meV}$. On the other hand, when the stacking chiralities are opposite ( $\alpha=+$ and $\left.\alpha^{\prime}=-\right)$, the total valley Chern number of the two bands equals to -2 at $\left|V_{\perp}\right|=0$, and remains unchanged for $\left|V_{\perp}\right| \lesssim 40 \mathrm{meV}$. Then the total valley Chern number of the two bands becomes +1 for 
TABLE I. The dependence of the Chern numbers on vertical electric potential for $(2+2)$-layer TMG.

\begin{tabular}{lccccccccccc}
\hline \hline$V_{\perp}$ & -60 & -48 & -36 & -24 & -12 & 0 & 12 & 24 & 36 & 48 & 60 \\
$C_{1++}^{K}$ & +1 & +1 & 0 & -2 & -3 & & +3 & +2 & 0 & -1 & -1 \\
$C_{2++}^{K}$ & +1 & +1 & +1 & +2 & +3 & & -3 & -2 & -1 & -1 & -1 \\
$C_{1+-}^{K}$ & 0 & 0 & +1 & +1 & 0 & 0 & 0 & +1 & +1 & 0 & 0 \\
$C_{2+-}^{K}$ & +1 & +1 & +1 & +1 & +2 & +2 & +2 & +1 & +1 & +1 & +1 \\
\hline \hline
\end{tabular}

TABLE II. The dependence of the Chern numbers on vertical electric potential for $(2+1)$-layer TMG.

\begin{tabular}{cccccccccccc}
\hline \hline$V_{\perp}$ & -40 & -32 & -24 & -16 & -8 & 0 & 8 & 16 & 24 & 32 & 40 \\
$C_{1+}^{K}$ & 0 & -1 & -1 & -1 & +2 & +1 & +2 & +2 & +2 & +2 & +2 \\
$C_{2+}^{K}$ & +2 & +2 & +2 & +2 & -1 & 0 & -1 & -1 & -1 & -1 & -1 \\
\hline \hline
\end{tabular}

$\left|V_{\perp}\right| \gtrsim 48 \mathrm{meV}$. Table I indicates that the topological phase of $(2+2)$-layer TMG is highly tunable by gate voltage, which is qualitatively in agreement with the results reported in Refs. [53,54]. Moreover, here we have also shown that it is sensitive to the stacking configurations.

In Table II we show $C_{n+}^{K}(n=1,2)$ versus $V_{\perp}$ for $(2+1)$-layer TMG at $\theta=1.05^{\circ}$, where the subscript " + " means that the bottom bilayer has + stacking chirality, and $n=1,2$ is the band index. When the vertical potential $V_{\perp}=0, C_{1+}^{K}=+1$ and $C_{2+}^{K}=0$, which is consistent with Eq. (8). Once a small positive or negative $V_{\perp} \sim 10 \mathrm{meV}$ is applied, $C_{1+}^{K}$ is changed to +2 and $C_{2+}^{K}$ becomes -1 . However, when $\left|V_{\perp}\right| \gtrsim 20 \mathrm{meV}$, the valley Chern numbers become highly dependent on the sign of $V_{\perp}$ due to the asymmetric stacking configuration of $(2+1)$-layer TMG. Thus, distinct topological phases could be realized by reversing the gate potentials.

\section{VERIFICATIONS USING AN EMPIRICAL TIGHT-BINDING (TB) MODEL}

The flat bands at the first magic angle (Fig. 2) and the Chern-number hierarchy [Eq. (8)] in TMG have been verified using a realistic microscopic tight-binding (TB) model. To be specific, the hopping parameter between two $p_{z}$ orbitals at different carbon sites $i$ and $j$ in the multilayer system is expressed in the Slater-Koster form,

$$
t(\mathbf{d})=V_{\sigma}\left(\frac{\mathbf{d} \cdot \hat{\mathbf{z}}}{d}\right)^{2}+V_{\pi}\left[1-\left(\frac{\mathbf{d} \cdot \hat{\mathbf{z}}}{d}\right)^{2}\right],
$$

where $V_{\sigma}=V_{\sigma}^{0} e^{-\left(r-d_{c}\right) / \delta_{0}}$ and $V_{\pi}=V_{\pi}^{0} e^{-\left(r-a_{0}\right) / \delta_{0}} \cdot \mathbf{d}=$ $\left(d_{x}, d_{y}, d_{z}\right)$ is the displacement vector between the two carbon sites. $a_{0}=a / \sqrt{3}=1.42 \AA, d_{c}=3.35 \AA$ is the interlayer distance in $A B$-stacked bilayer graphene, and $\delta_{0}=0.184 a . \quad V_{\sigma}^{0}=0.48 \mathrm{eV}$, and $V_{\pi}^{0}=-2.7 \mathrm{eV}$. The atomic corrugations at the interface between the $M$ layers
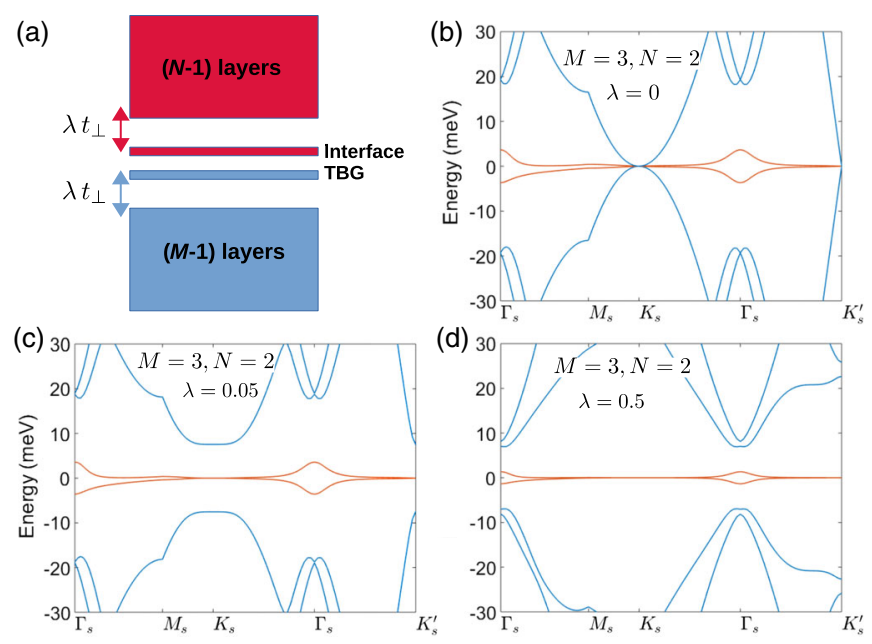

FIG. 5. (a) A schematic illustration of the TMG system. (b)-(d) The band structures of $(2+3)$-layer TMG at $\theta=1.05^{\circ}$. The coupling parameter between the interface and the top (bottom) multilayers has been rescaled by $\lambda$ : (b) $\lambda=0$, (c) $\lambda=0.05$, and (d) $\lambda=0.5$.

and the $N$ layers in $(M+N)$ TMG are modeled by Eq. (1), and their effects can be taken into account by plugging Eq. (1) into the hopping parameter shown in Eq. (9).

The band structures calculated using the Slater-Koster TB model at $\theta \approx 1.08^{\circ}$ for the $(2+2)$ TMG are shown in Fig. 6 . To be specific, the band structures of $(2+2) \mathrm{TMG}$ with opposite and the same stacking chiralities are shown in Figs. 6(a) and 6(c), respectively. It is evident that there are four low-energy bands (contributed by the two valleys) which are separated from the other high-energy bands, and the bandwidths are on the order of $10-15 \mathrm{meV}$, which are greater than those from the continuum model [see Fig. 2(a)]. This is because in the continuum model only the nearest-neighbor interlayer hopping is kept [see Eq. (A3)], which imposes a chiral symmetry to the Hamiltonian of Eq. (2). As argued in Appendix A, the chiral symmetry of Eq. (2) would pin the zeroth pseudo-LLs emerging from the twisted bilayer at the interface to zero energy, leading to almost vanishing bandwidth, as shown in Fig. 2. However, such a chiral symmetry is broken in the realistic SlaterKoster TB model, and the bandwidth of the flat bands is expected to be enhanced due to the presence of further neighbor interlayer hoppings.

In Figs. 6(b) and 6(d) we show the Wilson loops of the four low-energy bands of $(2+2)$ TMG at $\theta \approx 1.08^{\circ}$ calculated using the Slater-Koster TB model. Figure 6(b) [Fig. 6(d)] denotes the case with opposite (the same) stacking chiralities with the valley Chern number being \pm 2 (0). The topological equivalence between the band structures obtained by the more accurate tight-binding model as shown in Fig. 6 and by the continuum model as shown in Figs. 4(a) and 4(b) provides a strong supporting fact for the Chern number hierarchy given by Eq. (8). 

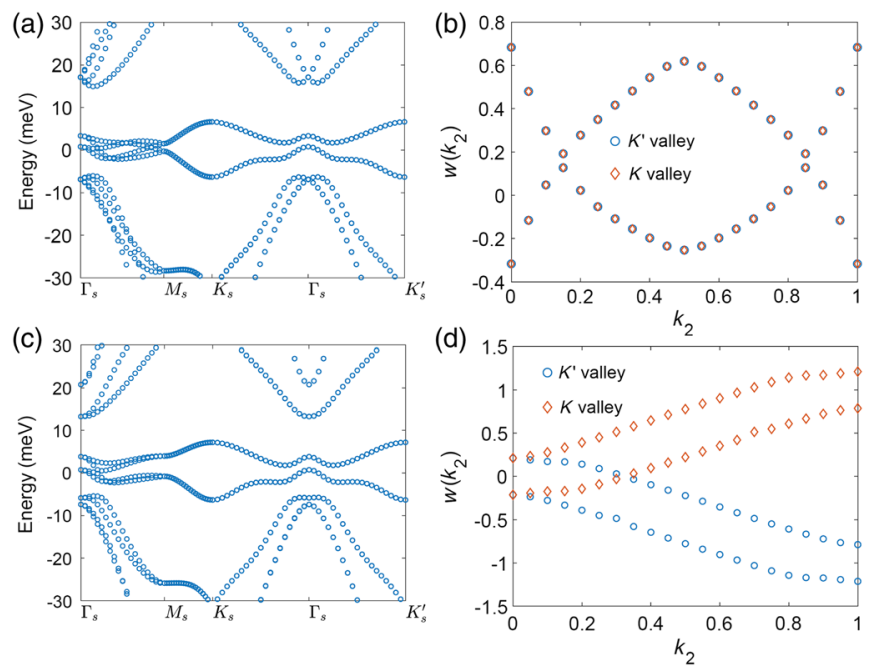

FIG. 6. The band structures of $(2+2)$ TMG at $\theta \approx 1.08^{\circ}$ (a) with opposite stacking chiralities and (c) with the same stacking chirality. The Wilson loops of $(2+2) \mathrm{TMG}$ at $\theta \approx 1.08^{\circ}$ (b) with opposite stacking chiralities and (d) with the same stacking chirality. The blue circles and red diamonds denote the Wilson loops of the $K$ and $K^{\prime}$ valleys.

\section{VALLEY-CONTRASTING ORBITAL MAGNETIZATIONS AND VALLEY-POLARIZED STATES}

The valley Chern numbers given by Eq. (8) imply opposite orbital magnetizations for the two monolayer valleys $K$ and $K^{\prime}$. In particular, according to the "modern theory" of orbital magnetizations [55-58], the orbital magnetization of the $(M+N)$-layer TMG for either the $K$ or $K^{\prime}$ valley can be expressed as

$$
\begin{aligned}
M_{z}= & \frac{e}{2 \hbar(2 \pi)^{2}} \sum_{n} \int_{\epsilon_{n \mathbf{k}} \leq \mu} d \mathbf{k} \operatorname{Im}\left\{\left\langle\partial_{\mathbf{k}} u_{n \mathbf{k}}\right|\right. \\
& \left.\times\left(H_{\mathbf{k}}+\epsilon_{n \mathbf{k}}-2 \mu\right)\left|\partial_{\mathbf{k}} u_{n \mathbf{k}}\right\rangle\right\}
\end{aligned}
$$

where $\epsilon_{n \mathbf{k}}$ and $\left|u_{n \mathbf{k}}\right\rangle$ are the eigenenergies and (the periodic part of) Bloch eigenstates of a $(M+N)$-layer TMG Hamiltonian (denoted by $H$ ) for either the $K$ or $K^{\prime}$ valley, $\mu$ is the chemical potential, and $H_{\mathbf{k}}=e^{-i \mathbf{k} \cdot \mathbf{r}} H e^{i \mathbf{k} \cdot \mathbf{r}}$. Since the $K$ and $K^{\prime}$ valleys are transformed to each other by a time-reversal operation, it is naturally expected that the valley-contrasting Chern numbers shown in Eq. (8) would lead to opposite orbital magnetization for the two valleys. The valley-contrasting orbital magnetizations further suggest that when the valley degeneracy is lifted by external magnetic fields or by Coulomb interactions, a valleypolarized state with nonvanishing or even quantized anomalous Hall conductivity will be generated due to the nonzero valley Chern numbers.

We have exploited this idea using the continuum model given by Eq. (2) with the interlayer hopping modeled by Eq. (7). To be explicit, we consider the case of $(2+2)$-layer
TMG at the first magic angle $\theta=1.05^{\circ}$. We have calculated the orbital magnetizations $\left(M_{z}\right)$ of the two low-energy bands for the $K$ valley using the Hamiltonian Eq. (2) with the interlayer hopping modeled by Eq. (7). The dependence of $M_{z}$ on the chemical potential $\mu$ is plotted in Fig. 7(a). The red diamonds in Fig. 7(a) represent the situation that the bottom bilayer and the top bilayer have opposite stacking chiralities with the valley Chern number \pm 2 [see Eq. (8)]. In this case the magnitude of $M_{z}$ is large, which is on the order of $10 \mu_{B}$ per moiré supercell when the two flat bands are completely filled. The large orbital magnetization is a manifestation of the band topology on the moire pattern. In particular, the nonvanishing Chern number of the $K$ or $K^{\prime}$ valley implies that the ground state at a given filling would possess chiral current loops. The characteristic radius of the current loop is on the order of the moire length scale $L_{s}$, which is associated with large orbital angular momentum $L_{z} \sim \mathbf{r} \times \mathbf{p}$, with $|\mathbf{p}| \sim \hbar K$ and $|\mathbf{r}| \sim L_{s} \gg a$. Therefore, the orbital magnetization generated by the current loops on the moiré scale is expected to be much greater than that on the microscopic lattice scale. We also note that $M_{z}$ increases almost linearly with $\mu$ when $\mu$ is in the gap above the two flat bands, which is a signature of the nonvanishing Chern number [57]. On the other hand, the blue circles in Fig. 7(a) denote the case that the bottom bilayer and the top bilayer have the same stacking chirality
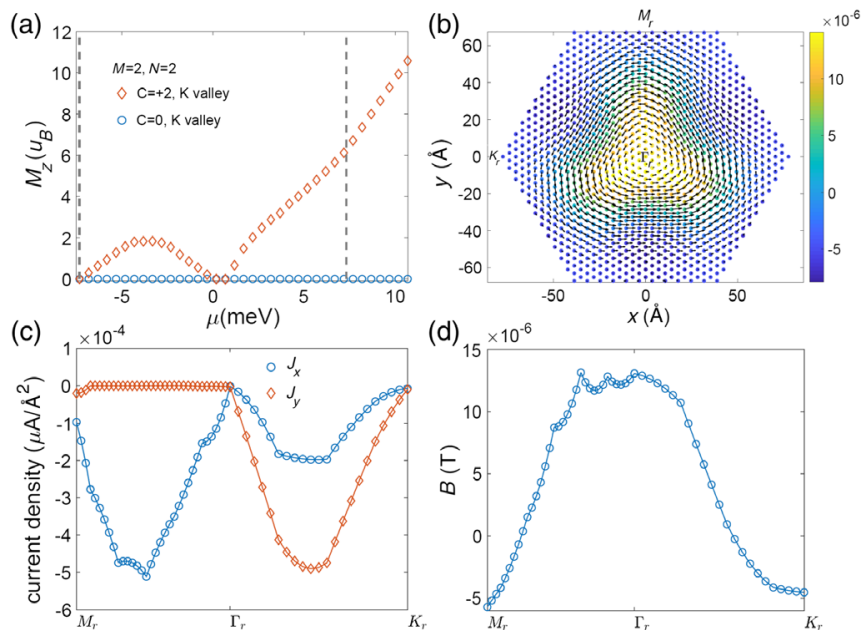

FIG. 7. (a) The orbital magnetization $M_{z}$ of the two flat bands of the $K$ valley for $(2+2)$-layer twisted graphene at the first magic angle. The red diamonds (blue circles) represent the situation that the stacking chiralities are opposite (the same), with the Chern number $+2(0)$. The horizontal axis is the chemical potential $\mu$. The vertical dashed lines mark the bulk band edges of the two low-energy flat bands. (b) The real-space distributions of the current density (black arrows) and the currentinduced magnetic field (color coding) within the moiré supercell. The $A A$ region is centered at the origin. (c) Current densities plotted along the real-space path $M_{r}-\Gamma_{r}-K_{r}$, where the $M_{r}$, $\Gamma_{r}$, and $K_{r}$ points are marked in (b). (d) The local magnetic field plotted along $M_{r}-\Gamma_{r}-K_{r}$. 
with vanishing valley Chern number. In this case, the orbital magnetization vanishes identically for any chemical potential due to the $C_{2 x}$ symmetry of the system. It is worth noting again that the orbital magnetization plotted in Fig. 7(a) is for the $K$ valley. The orbital magnetization for the $K^{\prime}$ valley is just opposite to that of the $K$ valley.

The large orbital magnetizations imply that the valley degeneracy of the system can be easily lifted by a weak external magnetic field or by spontaneous valley symmetry breaking from Coulomb interactions, leading to a valleypolarized (quantum) anomalous Hall state. A rough estimate reveals that a magnetic field of $2 \mathrm{~T}$ would give rise to an orbital (or valley) Zeeman splitting $\sim 2 \mathrm{meV}$ (15\% of the bandwidth), which would lead to a state with considerable valley polarization and anomalous Hall effect. Such a valleypolarized anomalous Hall state is expected to possess chiral current loops, which are responsible for the large orbital magnetization shown in Fig. 7(a). Here we assume that the $K$ valley is $100 \%$ polarized either due to the presence of an external magnetic field or due to spontaneous valley symmetry breaking from Coulomb interactions, and we calculate the local current density $\mathbf{J}(\mathbf{r})$ and the current-induced local magnetic field $B(\mathbf{r})$ with the two flat bands of the $K$ valley being completely filled. In Fig. 7(b) we show the real-space distributions of $\mathbf{J}(\mathbf{r})$ and $B(\mathbf{r})$ within the moiré Wigner-Seitz cell for the $(2+2)$-layer TMG with opposite stacking chiralities, and with $100 \%$ valley polarization. The small filled circles in Fig. 7(b) represent the discretized real-space positions, with the color coding denoting the strength of the local magnetic field in units of Tesla. The black arrows represent the local current densities whose amplitudes are proportional to the lengths of the arrows. Clearly the valleypolarized ground state possesses chiral current loops circulating around the $A A$ region. These circulating current loops further generate magnetic fields in the $A A$ region with the magnitude $\sim 10^{-5} \mathrm{~T}$, which may be a strong experimental evidence for the nonvanishing valley Chern number in $(2+2)$-layer TMG with opposite stacking chiralties.

In Fig. 7(c) we plot the current densities $J_{x}$ (blue circles) and $J_{y}$ (red diamonds) along the real-space path $M_{r}-\Gamma_{r}-K_{r}$ in units of $\mu \mathrm{A} / \AA^{2}$, where the $M_{r}, \Gamma_{r}$, and $K_{r}$ points are marked in Fig. 7(b). It is interesting to note that $J_{y}$ almost vanishes identically along the $M_{r}-\Gamma_{r}$ path due to the winding pattern of the current. The maximal magnitude of the current density $\sim 6 \times 10^{-4} \mu \mathrm{A} / \AA^{2}$. In Fig. 7(d) we show the local magnetic field plotted along the $M_{r}-\Gamma_{r}-K_{r}$ path. Clearly the magnetic field has a peak centered at $\Gamma_{r}$ (the $A A$ point) with the magnitude $\sim 10^{-5} \mathrm{~T}$. The details of the computation of the current densities and local magnetic fields are presented in Appendix C.

\section{DISCUSSION}

The results presented above are based on the simplified effective Hamiltonian Eq. (2), which assumes that the two valleys are decoupled from each other. This is an excellent approximation for the low-energy states at small twist angles, which has been widely adopted in the literature. However, even though the intervalley coupling becomes exponentially small at small twist angles, it is still present. Physically the intervalley coupling tends to mix the states of the two valleys, which may suppress the topological character of each valley. On the other hand, in reality the electrons' Coulomb interactions also need to be taken into account. In particular, the exchange part of the Coulomb interactions tends to split the two otherwise degenerate valleys, leading to a valley-polarized state. Thus the exchange splitting between the two valleys competes with the intervalley coupling. Given that the intervalley coupling is vanishingly small for small twist angles, we expect that the valley exchange interactions would dominate. In addition to the exchange interactions, the Hartree part of the Coulomb interactions may also be significant in modifying the band structures of the TMG system. This is because the Coulomb interactions may acquire valley-dependent form factors while being projected onto the low-energy bands [54]. Certainly a more thorough study is required in order to fully understand the effects of Coulomb interactions in the TMG system, and we leave it for future work.

Before concluding, we also would like to comment on the effects of impurities on the topological valley states. We consider impurity potentials which decay exponentially in real space, with a characteristic length scale $r_{0}$. The Fourier transform of the impurity potential would have a natural cutoff $q_{c} \sim \pi / r_{0}$ in the momentum space; i.e., the Fourier components for $q \gtrsim q_{c}$ can be neglected. If $r_{0}$ is on the order of the moiré length scale, then $q_{c}$ is comparable to the size of the moiree $\mathrm{BZ}$, and the impurity potential would only couple the low-energy states of TMG within the same valley. In this case, the topological features associated with each valley would remain robust. On the other hand, if $r_{0}$ is much smaller than the moiré length scale, say, on the order of microscopic lattice constant, then the impurity potential may lead to significant intervalley scatterings, which may locally destroy the current density and impede the topological properties associated with each valley. However, such an impurity effect should be perturbative in terms of the impurity concentration and impurity potential strength.

\section{SUMMARY}

To summarize,we have studied the electronic structures and topological properties of the $(M+N)$-layer TMG system. We have proposed that, with the chiral approximation, there always exist two low-energy bands whose bandwidths become minimally small at the magic angle of TBG. We have further shown that the two flat bands in the TMG system are topologically nontrivial and exhibit a Chern-number hierarchy. In particular, when the stacking chiralities of the $M$ layers and the $N$ layers are opposite, the total Chern number of the two low-energy bands for each 
valley equals $\pm(M+N-2)$ (per spin). On the other hand, if the stacking chiralities of the $M$ layers and the $N$ layers are the same, then the total Chern number of the two lowenergy bands for each valley is $\pm(M-N)$ (per spin). The nonvanishing valley Chern numbers are associated with large and valley-contrasting orbital magnetizations along the $z$ direction, which implies that the valley degeneracy can be lifted by weak external magnetic fields or by Coulomb interactions, leading to a valley-polarized anomalous Hall state. Such a valley-polarized state is associated with chiral current loops circulating around the $A A$ region, which generates local magnetic fields peaked at the $A A$ region. The local magnetic fields induced by the chiral current loops may be a robust experimental signature of the valley-polarized state with nonvanishing Chern number. Our work is a crucial step forward in understanding the electronic properties of twisted multilayer graphene. The universal magic angles and the Chern-number hierarchy proposed in this work make the TMG system a perfect platform to study the interplay between electrons' Coulomb correlations and nontrivial band topology.

\section{ACKNOWLEDGMENTS}

J.L. and X.D. acknowledge financial support from the Hong Kong Research Grants Council (Project No. GRF16300918). We thank Hongming Weng for invaluable discussions.

Note added.-Recently, we became aware of Refs. [54, $59,60]$. In the first work, the electronic structures, superconductivity, and the correlated insulating phase are discussed for twisted double bilayer graphene with the same stacking chirality ( $A B-A B$ stacking). In the second one, the band structures and valley Chern numbers of twisted double bilayer graphene with different stacking chiralities are discussed. In the last one, the effect of twists on the electronic structure of infinite stacks of graphene layers were studied.

\section{APPENDIX A: FLAT BANDS AND MAGIC ANGLES IN THE TMG SYSTEM}

In this appendix, we explain the origin of the flat bands and universal magic angles in the $(M+N)$-layer TMG system. After some gauge transformations, the constant wave vectors $\mathbf{K}_{M}, \mathbf{K}_{N}$ in Eq. (3) can be removed. Taking the case of the $(2+2)$-layer TMG with the same stacking chiralities as an example, the effective Hamiltonian is explicitly written as (after the gauge transformation)

$$
H_{+,+}^{K}(2+2)=\left(\begin{array}{cccc}
h_{0}(\hat{\mathbf{k}}) & h_{+} & 0 & 0 \\
h_{+}^{\dagger} & h_{0}(\hat{\mathbf{k}}) & U(\mathbf{r}) & 0 \\
0 & U^{\dagger}(\mathbf{r}) & h_{0}(\hat{\mathbf{k}}) & h_{+} \\
0 & 0 & h_{+}^{\dagger} & h_{0}(\hat{\mathbf{k}})
\end{array}\right) \text {, }
$$

where $h_{0}(\hat{\mathbf{k}})=-\hbar v_{F} \hat{\mathbf{k}} \cdot \boldsymbol{\sigma}$, and $h_{\alpha}$ and $U$ are defined in Eqs. (4) and (5) of the main text. Then we make the following unitary transformation to the basis functions of Eq. (A1) [and more generally, to those of Eq. (2) of the main text]:

$$
\begin{aligned}
& \left|\tilde{\psi}_{M s}\right\rangle=\frac{1}{2}\left(\left|\psi_{M s}\right\rangle+i\left|\psi_{(M+1) s}\right\rangle\right), \\
& \left|\tilde{\psi}_{(M+1) s}\right\rangle=\frac{1}{2}\left(\left|\psi_{M s}\right\rangle-i\left|\psi_{(M+1) s}\right\rangle\right), \\
& \left|\tilde{\psi}_{l s}\right\rangle=\left|\psi_{l s}\right\rangle, \quad \text { if } l \neq M, M+1,
\end{aligned}
$$

where $\left|\psi_{l s}\right\rangle$ denotes the Bloch state at the $K$ point from the $l$ th layer and the $s$ sublattice. Applying the unitary transformation Eq. (A2) to Eq. (A1) (letting $M=2$ ), then expanding the interlayer coupling term $U(\mathbf{r})$ to the linear order of $r / L_{s}$, Eq. (A1) becomes

$$
\tilde{H}_{+,+}^{K}(2+2)=\left(\begin{array}{cccc}
h_{0}(\hat{\mathbf{k}}) & \frac{h_{+}}{\sqrt{2}} & \frac{h_{+}}{\sqrt{2}} & 0 \\
\frac{h_{+}^{\dagger}}{\sqrt{2}} & h_{0}\left(\hat{\mathbf{k}}-\frac{e}{\hbar} \mathbf{A}\right) & -3 i u_{0} & -i \frac{h_{+}}{\sqrt{2}} \\
\frac{h_{+}^{\dagger}}{\sqrt{2}} & 3 i u_{0} & h_{0}\left(\hat{\mathbf{k}}+\frac{e}{\hbar} \mathbf{A}\right) & i \frac{h_{+}}{\sqrt{2}} \\
0 & -i \frac{h_{+}^{\dagger}}{\sqrt{2}} & i \frac{h_{+}^{\dagger}}{\sqrt{2}} & h_{0}(\hat{\mathbf{k}})
\end{array}\right),
$$

where the pseudovector potential $\mathbf{A}=\left(2 \pi u_{0}^{\prime}\right) /\left(L_{s} e v_{F}\right)$ $(y,-x)[18]$. Note that the diagonal blocks $h_{0}[\hat{\mathbf{k}} \pm(e / \hbar) \mathbf{A}]=$ $-\hbar v_{F}(\hat{\mathbf{k}} \pm e \mathbf{A} / \hbar)$ are equivalent to the Dirac fermions coupled with opposite pseudomagnetic fields, which would generate pseudo-LLs of opposite Chern numbers \pm 1 [18].
In particular, the two zeroth pseudo-LLs have opposite sublattice polarizations; thus the intrasublattice coupling term $\pm 3 i u_{0}$ in Eq. (A3) vanishes in the subspace of the zeroth pseudo-LLs [18]. Therefore, if we rewrite Eq. (A3) in a mixed basis consisting of the Dirac states from the first and fourth 
layers, and the zeroth pseudo-LLs from the second and third layers, then $\tilde{H}_{+,+}^{K}(2+2)$ becomes

$$
\tilde{H}_{+,+}^{K}(2+2) \approx\left(\begin{array}{cc}
h_{b} & 0 \\
0 & h_{t}
\end{array}\right) .
$$

Each element of Eq. (A4) is a $3 \times 3$ matrix. In particular,

$$
h_{b}=\left(\begin{array}{ccc}
0 & \hbar v_{F}\left(\hat{k}_{x}+i \hat{k}_{y}\right) & 0 \\
\hbar v_{F}\left(\hat{k}_{x}-i \hat{k}_{y}\right) & 0 & t_{\perp} \eta(k) \\
0 & t_{\perp} \eta^{*}(k) & 0
\end{array}\right)
$$

and

$$
h_{t}=\left(\begin{array}{ccc}
0 & i t_{\perp} \eta(k) & 0 \\
-i t_{\perp} \eta^{*}(k) & 0 & \hbar v_{F}\left(\hat{k}_{x}+i \hat{k}_{y}\right) \\
0 & \hbar v_{F}\left(\hat{k}_{x}-i \hat{k}_{y}\right) & 0
\end{array}\right) \text {, }
$$

where $\eta(k)$ denotes the coupling between the zeroth pseudoLL and the Dirac states of the first (fourth) layer, which can be expressed as some integral over the eigenfunctions of the zeroth pseudo-LLs and the Bloch functions, and $k$ is the index labeling the degenerate states within the zeroth pseudo LL. Note that we have dropped the higher pseudo-LLs in Eq. (A4).

The diagonalizations of Eqs. (A6) and (A5) would always yield two decoupled zero modes at any $k$. These two zero modes originate from the two zeroth pseudo-LLs contributed by the $M$ th and $(M+1)$ th twisted bilayer $[M=2$ for Eq. (A4)], and they stay at zero energy even after being coupled with the other layers due to the chiral symmetry of the effective Hamiltonian of TMG: all the matrix elements in Eqs. (2), (A1)-(A4) are intersublattice. As a consequence, if we apply the gauge transformation such that all the basis functions at the $B$ sublattice change sign, then the total Hamiltonian and eigenenergies would change sign as well. However, the eigenenergies are supposed to be invariant under such a gauge transformation, which thus enforces that both $E(\mathbf{k})$ and $-E(\mathbf{k})$ have to be the eigenenergies of the Hamiltonian. Therefore, a zero mode would stay at zero energy as long as the chiral symmetry is preserved. A similar argument applies to any $(M+N)$-layer TMG systems with either opposite or the same stacking chiralities. As long as the chiral symmetry is preserved, the zeroth pseudo-LLs emerging from the interface between the $M$ layers and $N$ layers would be pinned to zero energy.

On the other hand, it is well known that at the magic angles of TBG the bandwidth of the two low-energy bands for each valley is minimal. From the perspective of the pseudo-LLs [18], it means that at the magic angles, the states within the zeroth pseudo-LLs are minimally coupled with each other (and to the higher pseudo-LLs); thus they are almost exactly flat. As discussed above, by virtue of the chiral symmetry, these zeroth pseudo-LLs that are maximally flat at the magic angles would remain flat even after being coupled with the other layers in the TMG systems. It follows that the magic angles in TBG should be universal in the TMG systems by virtue of the chiral symmetry in Eq. (2).

\section{APPENDIX B: DERIVATIONS OF THE CHERN-NUMBER HIERARCHY}

In this appendix, we mathematically prove the Chernnumber hierarchy given by Eq. (8). As discussed in Sec. III A, the $(M+N)$-layer TMG system can be decomposed into three subsystems: the TBG at the interface, the $(M-1)$ layers below the interface, and the $(N-1)$ layers above the interface. This is schematically shown in Fig. 5(a). In graphene multilayers with + stacking chirality, the $B$ sublattice of the $n$th layer is strongly coupled with the $A$ sublattice of the $(n+1)$ th layer, forming pairs of bounding and antibounding states, leaving the $A$ sublattice of the first layer and the $B$ sublattice of the $N$ th layer ( $N$ is the number of layers) as two low-energy degrees of freedom. Similarly, in graphene multlayers with - stacking chirality, the $B$ sublattice of the first layer and the $A$ sublattice of the $N$ th layer would be the low-energy degrees of freedom. The low-energy effective Hamiltonians of the $(M-1)$-layer graphene around the $K$ valley with $\alpha$ ( $\alpha= \pm 1)$ stacking chirality can be obtained by straightforward $(M-1)$ th order perturbation theory [61], which is expressed as

$H_{\text {eff }}^{\alpha}(M-1)=(-1)^{M-2}\left(\begin{array}{cc}0 & \frac{\left[\hbar v_{F}\left(k_{x}+i \alpha k_{y}\right)\right]^{M-1}}{t_{\perp}^{M-2}} \\ \frac{\left[\hbar v_{F}\left(k_{x}-i \alpha k_{y}\right)\right]^{M-1}}{t_{\perp}^{M-2}} & 0\end{array}\right)$,

where $t_{\perp}$ is the interlayer hopping within the $(M-1)$ layers. Equation (B1) is in the basis of $|1, A\rangle(|1, B\rangle)$ and $|M-1, B\rangle(|M-1, A\rangle)$ if the $(M-1)$ layers have $+(-)$ stacking chirality. On the other hand, there are two nearly flat bands at the magic angle contributed by the interface TBG. Around the $K_{s}$ and $K_{s}^{\prime}$ points these two flat bands are equivalent to zeroth pseudo-LLs, and possess opposite sublattice polarizations, as argued in Ref. [18]. Let us first assume the coupling between the $(M-1)$ layers and the interface TBG is small, then the low-energy effective Hamiltonian around the $K_{s}$ point can be expressed as

$$
H_{K_{s}}^{\alpha}(\mathbf{k})=\left(\begin{array}{ccc}
0 & \frac{\left(k_{x}+i \alpha k_{y}\right)^{M-1}}{m_{M-1}} & 0 \\
\frac{\left(k_{x}-i \alpha k_{y}\right)^{M-1}}{m_{M-1}} & 0 & t_{\mathrm{eff}} \\
0 & t_{\mathrm{eff}} & 0
\end{array}\right),
$$


where

$$
m_{M-1}=\frac{t_{\perp}^{M-2}}{(-1)^{M-2}\left(\hbar v_{F}\right)^{M-1}},
$$

and $t_{\text {eff }}$ is the low-energy effective coupling between the states of the $(M-1)$ layers and the flat bands from the interface TBG. In particular, if the stacking chirality is + , $t_{\text {eff }}$ represents the coupling between the Bloch states from the $B$ sublattice of the $(M-1)$ th layer and one of the two flat bands with $A$ sublattice polarization contributed by the interface TBG. If the stacking chirality is - , then $t_{\text {eff }}$ represents the coupling between the Bloch states from the $A$ sublattice of the $(M-1)$ th layer and one of the two flat bands with $B$ sublattice polarization contributed by the interface TBG. Note that although $\mathbf{k}$ in Eq. (B1) denotes the $\mathbf{k}$ point in the original primitive-cell $\mathrm{BZ}, \mathbf{k}$ in Eq. (B2) represents the $\mathbf{k}$ point in the moire supercell BZ. This is because the form of the low-energy effective Hamiltonian [Eq. (B1)] is unchanged after the BZ folding.

Similarly, one can write down the low-energy effective Hamiltonian around the $K_{s}^{\prime}$ point, which consists of the two low-energy states from the $(N-1)$ layers above the TBG interface and one of the two flat bands from the TBG interface. More explicitly, it can be expressed as

$$
H_{K_{s}^{\prime}}^{\alpha^{\prime}}(\mathbf{k})=\left(\begin{array}{ccc}
0 & \frac{\left(k_{x}+i \alpha^{\prime} k_{y}\right)^{N-1}}{m_{N-1}} & t_{\mathrm{eff}}^{*} \\
\frac{\left(k_{x}-i \alpha^{\prime} k_{y}\right)^{N-1}}{m_{N-1}} & 0 & 0 \\
t_{\mathrm{eff}} & 0 & 0
\end{array}\right) \text {, }
$$

where $\alpha^{\prime}= \pm$ represents the stacking chirality of the $N$ layers and $m_{N-1}$ is given by Eq. (B3).

Both Eqs. (B2) and (B4) can be solved analytically. The eigenenergies of Eq. (B2) are expressed as

$$
\begin{aligned}
\epsilon_{1 \mathbf{k}}(M-1) & =-\sqrt{m_{M-1}^{2} k^{2 M-2}+\left|t_{\mathrm{eff}}\right|^{2}}, \\
\epsilon_{2 \mathbf{k}} & =0, \\
\epsilon_{3 \mathbf{k}}(M-1) & =\sqrt{m_{M-1}^{2} k^{2 M-2}+\left|t_{\mathrm{eff}}\right|^{2}} .
\end{aligned}
$$

The eigenenergies of Eq. (B4) have exactly the same analytic expression but with $(M-1)$ replaced by $(N-1)$. The eigenstates of $H_{K_{s}}^{\alpha}(\mathbf{k})$ are expressed as

$$
\begin{aligned}
\left|\psi_{1 \mathbf{k}}^{M-1}\right\rangle & =\left(\frac{\left(k_{x}+i \alpha k_{y}\right)^{M-1}}{\sqrt{2} g_{M-1}(|\mathbf{k}|)},-\frac{1}{\sqrt{2}}, \frac{\tilde{t}_{\mathrm{eff}}}{\sqrt{2} g_{M-1}(|\mathbf{k}|)}\right)^{T}, \\
\left|\psi_{2 \mathbf{k}}^{M-1}\right\rangle & =\left(\frac{-\tilde{t}_{\mathrm{eff}} e^{i \alpha \theta_{\mathbf{k}}(M-1)}}{g_{M-1}(\mathbf{k})}, 0, \frac{|\mathbf{k}|^{(M-1)}}{g_{M-1}(\mathbf{k})}\right)^{T}, \\
\left|\psi_{3 \mathbf{k}}^{M-1}\right\rangle & =\left(\frac{\left(k_{x}+i \alpha k_{y}\right)^{M-1}}{\sqrt{2} g_{M-1}(|\mathbf{k}|)}, \frac{1}{\sqrt{2}}, \frac{\tilde{t}_{\mathrm{eff}}}{\sqrt{2} g_{M-1}(|\mathbf{k}|)}\right)^{T},
\end{aligned}
$$

where $g_{M-1}(|\mathbf{k}|)=\sqrt{\tilde{t}_{\text {eff }}^{2}+|\mathbf{k}|^{2 M-2}}, \tilde{t}_{\text {eff }}=t_{\text {eff }} / m_{M-1}$, and $\theta_{\mathbf{k}}=\arctan \left(k_{y} / k_{x}\right)$. The eigenstates of $H_{K_{s}^{\prime}}^{\alpha^{\prime}}(\mathbf{k})$ are expressed as

$\left|\psi_{1 \mathbf{k}}^{N-1}\right\rangle=\left(-\frac{1}{\sqrt{2}}, \frac{\left(k_{x}-i \alpha^{\prime} k_{y}\right)^{N-1}}{\sqrt{2} g_{N-1}(|\mathbf{k}|)}, \frac{\tilde{t}_{\mathrm{eff}}}{\sqrt{2} g_{N-1}(|\mathbf{k}|)}\right)^{T}$,

$\left|\psi_{2 \mathbf{k}}^{N-1}\right\rangle=\left(0,-\frac{\tilde{t}_{\mathrm{eff}} e^{-i \alpha^{\prime} \theta_{\mathbf{k}}(N-1)}}{g_{N-1}(\mathbf{k})}, \frac{|\mathbf{k}|^{(N-1)}}{g_{N-1}(\mathbf{k})}\right)^{T}$,

$\left|\psi_{3 \mathbf{k}}^{N-1}\right\rangle=\left(\frac{1}{\sqrt{2}}, \frac{\left(k_{x}-i \alpha^{\prime} k_{y}\right)^{N-1}}{\sqrt{2} g_{N-1}(|\mathbf{k}|)}, \frac{\tilde{t}_{\mathrm{eff}}}{\sqrt{2} g_{N-1}(|\mathbf{k}|)}\right)^{T}$.

Given that $\left(k_{x} \pm \alpha k_{y}\right)^{M-1}$ can be rewritten as $|\mathbf{k}|^{M-1} e^{i \pm \alpha \theta_{\mathbf{k}}(M-1)}$, it is straightforward to calculate the Berry connections of the valence states and the conduction states. For the states around the $K_{s}$ point [Eq. (B6)], it turns out that

$A_{1 \theta_{\mathbf{k}}}^{M-1}=i\left\langle\psi_{1 \mathbf{k}}^{M-1} \mid \partial_{\theta_{\mathbf{k}}} \psi_{1 \mathbf{k}}^{M-1}\right\rangle=-\frac{\alpha(M-1)|\mathbf{k}|^{2 M-2}}{2 \tilde{t}_{\mathrm{eff}}^{2}+2|\mathbf{k}|^{2 M-2}}$,

and $A_{3 \theta_{\mathbf{k}}}^{M-1}=A_{1 \theta_{\mathbf{k}}}^{M-1}$. For the states around the $K_{s}^{\prime}$ point, we have

$$
A_{1 \theta_{\mathbf{k}}}^{N-1}=i\left\langle\psi_{1 \mathbf{k}}^{N-1} \mid \partial_{\theta_{\mathbf{k}}} \psi_{1 \mathbf{k}}^{N-1}\right\rangle=\frac{\alpha^{\prime}(N-1)|\mathbf{k}|^{2 N-2}}{2 \tilde{t}_{\mathrm{eff}}^{2}+2|\mathbf{k}|^{2 N-2}},
$$

and $A_{3 \theta_{\mathbf{k}}}^{N-1}=A_{1 \theta_{\mathbf{k}}}^{N-1}$. It is interesting to note that the valence and conduction states $\left|\psi_{1 \mathbf{k}}\right\rangle$ and $\left|\psi_{3 \mathbf{k}}\right\rangle$ have the same Berry connections; therefore, they have the same Berry phase by virtue of the chiral symmetry of Eqs. (B2) and (B4). Taking the limit $\tilde{t}_{\text {eff }} \rightarrow 0$, from Eqs. (B8) and (B9) it follows that $A_{1 \theta_{\mathbf{k}}}^{M-1}=A_{3 \theta_{\mathbf{k}}}^{M-1} \rightarrow-\alpha(M-1) / 2$ and $A_{1 \theta_{\mathbf{k}}}^{N-1}=A_{3 \theta_{\mathbf{k}}}^{N-1} \rightarrow$ $\alpha^{\prime}(N-1) / 2$. Therefore, around the $K_{s}\left(K_{s}^{\prime}\right)$ point, the sum of the Berry fluxes of the conduction and the valence bands equals $-\alpha(M-1)\left[\alpha^{\prime}(N-1)\right]$. Then the total Chern number of the conduction and the valence bands equal $-\alpha(M-1)+\alpha^{\prime}(N-1)$. The Chern number of the two flat bands $\left(\left|\psi_{2 \mathbf{k}}\right\rangle\right)$ must cancel the total Chern number of the valence and conduction bands $\left(\left|\psi_{1 \mathbf{k}}\right\rangle\right.$ and $\left.\left|\psi_{3 \mathbf{k}}\right\rangle\right)$; it follows that the total Chern number of the two flat bands equals $\alpha(M-1)-\alpha^{\prime}(N-1)$ for the $K$ valley. The total Chern number of the two flat bands of the $K^{\prime}$ valley is just opposite to that of the $K$ valley; thus Eq. (8) has been proved.

\section{APPENDIX C: CALCULATING THE LOCAL CHARGE CURRENT USING THE CONTINUUM MODEL}

The current density operator at a position $\mathbf{r}$ is expressed as 


$$
\hat{\mathbf{J}}(\mathbf{r})=e \hat{\rho}(\mathbf{r}) \hat{\mathbf{v}},
$$

where $\hat{\rho}(\mathbf{r})$ is the local density operator at $\mathbf{r}$, and the velocity operator $\hat{\mathbf{v}}$ satisfies the Schördinger equation $-i \hbar \hat{\mathbf{v}}=[H(\mathbf{r}), \mathbf{r}]$. The Hamiltonian at $\mathbf{r}$ is given by Eq. (2), with $\mathbf{k}=-i \partial_{\mathbf{r}}$. Then it is straightforward to calculate the velocity operator by performing the commutator of $H(\mathbf{r})$ and $\mathbf{r}$. The expectation value of $\hat{\mathbf{J}}(\mathbf{r})$ then equals

$$
\langle\hat{\mathbf{J}}(\mathbf{r})\rangle=e \sum_{s \mathbf{G k}}\langle s \mathbf{G}, \mathbf{k}|\hat{\rho} \hat{\rho}(\mathbf{r}) \hat{\mathbf{v}}| s \mathbf{G}, \mathbf{k}\rangle,
$$

where $\hat{\rho}=\sum_{n \mathbf{k}}\left|\psi_{n \mathbf{k}}\right\rangle\left\langle\psi_{n \mathbf{k}}\right| \theta\left(\mu-\epsilon_{n \mathbf{k}}\right)$ is the density matrix at zero temperature with the chemical potential $\mu$, with $\left|\psi_{n \mathbf{k}}\right\rangle$ and $\epsilon_{n \mathbf{k}}$ being the $n$th eigenstate and eigenenergy of the Hamiltonian Eq. (2) at the $\mathbf{k}$ point in the moire BZ. $|s \mathbf{G}, \mathbf{k}\rangle$ is the plane-wave function, where $\mathbf{G}$ represents a reciprocal lattice vector of the moire cell, and $s$ is the index for the layer and sublattice degrees of freedom. To be more explicit, in the plane-wave basis, $\hat{\rho}, \rho(\mathbf{r})$, and $\hat{v}$ are expressed as

$$
\begin{gathered}
\left\langle s \mathbf{G}, \mathbf{k}|\hat{\rho}| s^{\prime} \mathbf{G}^{\prime}, \mathbf{k}\right\rangle=\sum_{n \mathbf{k}} C_{s \mathbf{G}, n}(\mathbf{k}) C_{s^{\prime} \mathbf{G}^{\prime}, n}^{*}(\mathbf{k}) \theta\left(\mu-\epsilon_{n \mathbf{k}}\right), \\
\left\langle s \mathbf{G}, \mathbf{k}|\hat{\rho}(\mathbf{r})| s^{\prime} \mathbf{G}^{\prime}, \mathbf{k}\right\rangle=\frac{1}{V} \delta_{s s^{\prime}} e^{-i \mathbf{G} \cdot \mathbf{r}} e^{i \mathbf{G}^{\prime} \cdot \mathbf{r}}, \\
\left\langle s \mathbf{G}, \mathbf{k}|\hat{\mathbf{v}}| s^{\prime} \mathbf{G}^{\prime}, \mathbf{k}\right\rangle=\left\langle s \mathbf{G}, \mathbf{k}\left|\frac{\partial H_{\mathbf{k}}}{\hbar \partial \mathbf{k}}\right| s^{\prime} \mathbf{G}^{\prime}, \mathbf{k}\right\rangle,
\end{gathered}
$$

where $C_{s \mathbf{G}, n}(\mathbf{k})$ is the plane-wave coefficient of the eigenstate $\left|\psi_{n \mathbf{k}}\right\rangle$; i.e., $\left|\psi_{n \mathbf{k}}\right\rangle=\sum_{s \mathbf{G}} C_{s \mathbf{G}, n}(\mathbf{k})|s \mathbf{G}, \mathbf{k}\rangle . V$ is the total volume of the system, and $H_{\mathbf{k}}=e^{-i \mathbf{k} \cdot \mathbf{r}} H e^{i \mathbf{k} \cdot \mathbf{r}}$, where the Hamiltonian $H$ is given by Eq. (2). Given the current density distribution $\mathbf{J}(\mathbf{r})$, the magnetic field $B(\mathbf{r})$ can be solved using Ampère's law, $\nabla \times \mathbf{B}(\mathbf{r})=\mu_{0} \mathbf{J}(\mathbf{r})$, where $\mu_{0}$ is the magnetic permeability of the vacuum.

[1] Y. Cao, V. Fatemi, A. Demir, S. Fang, S. L. Tomarken, J. Y. Luo, J. D. Sanchez-Yamagishi, K. Watanabe, T. Taniguchi, E. Kaxiras et al., Correlated Insulator Behaviour at HalfFilling in Magic-Angle Graphene Superlattices, Nature (London) 556, 80 (2018).

[2] A. L. Sharpe, E. J. Fox, A. W. Barnard, J. Finney, K. Watanabe, T. Taniguchi, M. A. Kastner, and D. Goldhaber-Gordon, Emergent Ferromagnetism Near Three-Quarters Filling in Twisted Bilayer Graphene, arXiv: 1901.03520.

[3] Y. Choi, J. Kemmer, Y. Peng, A. Thomson, H. Arora, R. Polski, Y. Zhang, H. Ren, J. Alicea, G. Refael et al., Imaging Electronic Correlations in Twisted Bilayer Graphene Near the Magic Angle, arXiv:1901.02997.
[4] A. Kerelsky, L. McGilly, D. M. Kennes, L. Xian, M. Yankowitz, S. Chen, K. Watanabe, T. Taniguchi, J. Hone, C. Dean et al., Magic Angle Spectroscopy, arXiv:1812 .08776 .

[5] E. Codecido, Q. Wang, R. Koester, S. Che, H. Tian, R. Lv, S. Tran, K. Watanabe, T. Taniguchi, F. Zhang et al., Correlated Insulating and Superconducting States in Twisted Bilayer Graphene Below the Magic Angle, arXiv:1902.05151.

[6] Y. Cao, V. Fatemi, S. Fang, K. Watanabe, T. Taniguchi, E. Kaxiras, and P. Jarillo-Herrero, Unconventional Superconductivity in Magic-Angle Graphene Superlattices, Nature (London) 556, 43 (2018).

[7] M. Yankowitz, S. Chen, H. Polshyn, Y. Zhang, K. Watanabe, T. Taniguchi, D. Graf, A. F. Young, and C. R. Dean, Tuning Superconductivity in Twisted Bilayer Graphene, Science 363, 1059 (2019).

[8] J. M. B. L. dos Santos, N. M. R. Peres, and A. H. C. Neto, Graphene Bilayer with a Twist: Electronic Structure, Phys. Rev. Lett. 99, 256802 (2007).

[9] R. Bistritzer and A. H. MacDonald, Moiré Bands in Twisted Double-Layer Graphene, Proc. Natl. Acad. Sci. U.S.A. 108, 12233 (2011).

[10] H. C. Po, L. Zou, A. Vishwanath, and T. Senthil, Origin of Mott Insulating Behavior and Superconductivity in Twisted Bilayer Graphene, Phys. Rev. X 8, 031089 (2018).

[11] N. F. Q. Yuan and L. Fu, Model for the Metal-Insulator Transition in Graphene Superlattices and Beyond, Phys. Rev. B 98, 045103 (2018).

[12] M. Koshino, N. F. Q. Yuan, T. Koretsune, M. Ochi, K. Kuroki, and L. Fu, Maximally Localized Wannier Orbitals and the Extended Hubbard Model for Twisted Bilayer Graphene, Phys. Rev. X 8, 031087 (2018).

[13] J. Kang and O. Vafek, Symmetry, Maximally Localized Wannier States, and a Low-Energy Model for Twisted Bilayer Graphene Narrow Bands, Phys. Rev. X 8, 031088 (2018).

[14] Z. Song, Z. Wang, W. Shi, G. Li, C. Fang, and B. A. Bernevig, All Magic Angles in Twisted Bilayer Graphene are Topological, Phys. Rev. Lett. 123, 036401 (2019).

[15] H. C. Po, L. Zou, T. Senthil, and A. Vishwanath, Faithful Tight-Binding Models and Fragile Topology of MagicAngle Bilayer Graphene, Phys. Rev. B 99, 195455 (2019).

[16] G. Tarnopolsky, A. Jura Kruchkov, and A. Vishwanath, Origin of Magic Angles in Twisted Bilayer Graphene, Phys. Rev. Lett. 122, 106405 (2019).

[17] H. K. Pal, S. Spitz, and M. Kindermann, Emergent Geometric Frustration and Flat Band in Moiré Bilayer Graphene, arXiv:1803.07060.

[18] J. Liu, J. Liu, and X. Dai, Pseudo Landau Level Representation of Twisted Bilayer Graphene: Band Topology and Implications on the Correlated Insulating Phase, Phys. Rev. B 99, 155415 (2019).

[19] B. Lian, F. Xie, and B. A. Bernevig, The Landau Level of Fragile Topology, arXiv:1811.11786.

[20] Y.-H. Zhang, D. Mao, and T. Senthil, Twisted Bilayer Graphene Aligned with Hexagonal Boron Nitride: Anomalous Hall Effect and a Lattice Model, arXiv:1901.08209.

[21] A. O. Sboychakov, A. V. Rozhkov, A. L. Rakhmanov, and F. Nori, Many-Body Effects in Twisted Bilayer Graphene at Low Twist Angles, Phys. Rev. B 100, 045111 (2019). 
[22] H. Isobe, N. F. Q. Yuan, and L. Fu, Unconventional Superconductivity and Density Waves in Twisted Bilayer Graphene, Phys. Rev. X 8, 041041 (2018).

[23] X. Y. Xu, K. T. Law, and P. A. Lee, Kekulé Valence Bond Order in an Extended Hubbard Model on the Honeycomb Lattice with Possible Applications to Twisted Bilayer Graphene, Phys. Rev. B 98, 121406(R) (2018).

[24] T. Huang, L. Zhang, and T. Ma, Antiferromagnetically Ordered Mott Insulator and $d+i d$ Superconductivity in Twisted Bilayer Graphene: A Quantum Monte Carlo Study, Sci. Bull. 64, 310 (2019).

[25] C.-C. Liu, L.-D. Zhang, W.-Q. Chen, and F. Yang, Chiral Spin Density Wave and $d+i d$ Superconductivity in the Magic-Angle-Twisted Bilayer Graphene, Phys. Rev. Lett. 121, 217001 (2018).

[26] L. Rademaker and P. Mellado, Charge-Transfer Insulation in Twisted Bilayer Graphene, Phys. Rev. B 98, 235158 (2018).

[27] J. W. F. Venderbos and R. M. Fernandes, Correlations and Electronic Order in a Two-Orbital Honeycomb Lattice Model for Twisted Bilayer Graphene, Phys. Rev. B 98, 245103 (2018).

[28] J. Kang and O. Vafek, Strong Coupling Phases of Partially Filled Twisted Bilayer Graphene Narrow Bands, Phys. Rev. Lett. 122, 246401 (2019).

[29] M. Xie and A. H. MacDonald, On the Nature of the Correlated Insulator States in Twisted Bilayer Graphene, arXiv:1812.04213.

[30] C.-M. Jian and C. Xu, Moire Mott Insulators Viewed as the Surface of Three Dimensional Symmetry Protected Topological Phases, arXiv:1810.03610.

[31] N. Bultinck, S. Chatterjee, and M. P. Zaletel, Anomalous Hall Ferromagnetism in Twisted Bilayer Graphene, arXiv: 1901.08110.

[32] C. Xu and L. Balents, Topological Superconductivity in Twisted Multilayer Graphene, Phys. Rev. Lett. 121, 087001 (2018).

[33] F. Wu, A. H. MacDonald, and I. Martin, Theory of PhononMediated Superconductivity in Twisted Bilayer Graphene, Phys. Rev. Lett. 121, 257001 (2018).

[34] X.-C. Wu, K. A. Pawlak, C.-M. Jian, and C. Xu, Emergent Superconductivity in the Weak Mott Insulator Phase of Bilayer Graphene Moiré Superlattice, arXiv:1805.06906.

[35] B. Lian, Z. Wang, and B. A. Bernevig, Twisted Bilayer Graphene: A Phonon Driven Superconductor, Phys. Rev. Lett. 122, 257002 (2019).

[36] V. Kozii, H. Isobe, J. W. F. Venderbos, and L. Fu, Nematic Superconductivity Stabilized by Density Wave Fluctuations: Application to Twisted Bilayer Graphene, Phys. Rev. B 99, 144507 (2019).

[37] F. Wu, Topological Chiral Superconductivity with Spontaneous Vortices and Supercurrent in Twisted Bilayer Graphene, Phys. Rev. B 99, 195114 (2019).

[38] B. Roy and V. Jurić, Unconventional Superconductivity in Nearly Flat Bands in Twisted Bilayer Graphene, Phys. Rev. B 99, 121407(R) (2019).

[39] J. Ahn, S. Park, and B.-J. Yang, Failure of Nielsen-Ninomiya Theorem and Fragile Topology in Two-Dimensional Systems with Space-Time Inversion Symmetry: Application to
Twisted Bilayer Graphene at Magic Angle, Phys. Rev. X 9, 021013 (2019).

[40] C. Shen, N. Li, S. Wang, Y. Zhao, J. Tang, J. Liu, J. Tian, Y. Chu, K. Watanabe, T. Taniguchi et al., Observation of Superconductivity with Tc Onset at $12 \mathrm{~K}$ in Electrically Tunable Twisted Double Bilayer Graphene, arXiv:1903 .06952 .

[41] X. Liu, Z. Hao, E. Khalaf, J. Y. Lee, K. Watanabe, T. Taniguchi, A. Vishwanath, and P. Kim, Spin-Polarized Correlated Insulator and Superconductor in Twisted Double Bilayer Graphene, arXiv:1903.08130.

[42] Y. Cao, D. Rodan-Legrain, O. Rubies-Bigord, J. M. Park, K. Watanabe, T. Taniguchi, and P. Jarillo-Herrero, Electric Field Tunable Correlated States and Magnetic Phase Transitions in Twisted Bilayer-Bilayer Graphene, arXiv: 1903.08596.

[43] G. Chen, L. Jiang, S. Wu, B. Lyu, H. Li, B. L. Chittari, K. Watanabe, T. Taniguchi, Z. Shi, J. Jung et al., Evidence of a Gate-Tunable Mott Insulator in a Trilayer Graphene Moiré Superlattice, Nat. Phys. 15, 237 (2019).

[44] G. Chen, A. L. Sharpe, G. Patrick, I. T. Rosen, E. Fox, L. Jiang, B. Lyu, H. Li, K. Watanabe, T. Taniguchi, J. Jung, Z. Shi, D. Goldhaber-Gordon, Y. Zhang, and W. Feng, Signatures of Gate-Tunable Superconductivity in Trilayer Graphene/Boron Nitride Moirè Superlattice, arXiv:1901.04621.

[45] G. Chen, A. L. Sharpe, E. J. Fox, Y.-H. Zhang, S. Wang, L. Jiang, B. Lyu, H. Li, K. Watanabe, T. Taniguchi et al., Tunable Correlated Chern Insulator and Ferromagnetism in Trilayer Graphene/Boron Nitride Moiré Superlattice, arXiv:1905.06535.

[46] N. R. Chebrolu, B. L. Chittari, and J. Jung, Flatbands in Twisted Bi-Bilayer Graphene, Phys. Rev. B 99, 235417 (2019).

[47] Unless otherwise specified, in the rest of the paper the Chern number refers to the Chern number for each physical spin species.

[48] J. M. B. L. dos Santos, N. M. R. Peres, and A. H. C. Neto, Continuum Model of the Twisted Graphene Bilayer, Phys. Rev. B 86, 155449 (2012).

[49] J.-K. Lee, S.-C. Lee, J.-P. Ahn, S.-C. Kim, J. I. B. Wilson, and P. John, The Growth of AA Graphite on (111) Diamond, J. Chem. Phys. 129, 234709 (2008).

[50] The band structures with opposite stacking chiralities are very similar to those shown in Fig. 2.

[51] The Dirac points of the $M$ graphene layers $K_{M}$ and of the $N$ graphene monolayers $K_{N}$ are, respectively, mapped to $K_{s}$ and $K_{s}^{\prime}$ points of the moiré Brillouin zone; see Fig. 1(b).

[52] J.-W. Rhim and B.-J. Yang, Classification of Flat Bands According to the Band-Crossing Singularity of Bloch Wave Functions, Phys. Rev. B 99, 045107 (2019).

[53] Y.-H. Zhang, D. Mao, Y. Cao, P. Jarillo-Herrero, and T. Senthil, Nearly Flat Chern Bands in Moiré Superlattices, Phys. Rev. B 99, 075127 (2019).

[54] J. Y. Lee, E. Khalaf, S. Liu, X. Liu, Z. Hao, P. Kim, and A. Vishwanath, Theory of Correlated Insulating Behaviour and Spin-Triplet Superconductivity in Twisted Double Bilayer Graphene, arXiv:1903.08685.

[55] T. Thonhauser, D. Ceresoli, D. Vanderbilt, and R. Resta, Orbital Magnetization in Periodic Insulators, Phys. Rev. Lett. 95, 137205 (2005). 
[56] D. Xiao, J. Shi, and Q. Niu, Berry Phase Correction to Electron Density of States in Solids, Phys. Rev. Lett. 95, 137204 (2005).

[57] D. Ceresoli, T. Thonhauser, D. Vanderbilt, and R. Resta, Orbital Magnetization in Crystalline Solids: Multi-Band Insulators, Chern Insulators, and Metals, Phys. Rev. B 74, 024408 (2006).

[58] J. Shi, G. Vignale, D. Xiao, and Q. Niu, Quantum Theory of Orbital Magnetization and Its Generalization to Interacting Systems, Phys. Rev. Lett. 99, 197202 (2007).
[59] M. Koshino, Band Structure and Topological Properties of Twisted Double Bilayer Graphene, Phys. Rev. B 99, 235406 (2019).

[60] T. Cea, N. R. Walet, and F. Guinea, Twists and the Electronic Structure of Graphitic Materials, Phys. Rev. B 99, 235406 (2019).

[61] H. Min and A.H. MacDonald, Electronic Structure of Multilayer Graphene, Prog. Theor. Phys. Suppl. 176, 227 (2008). 